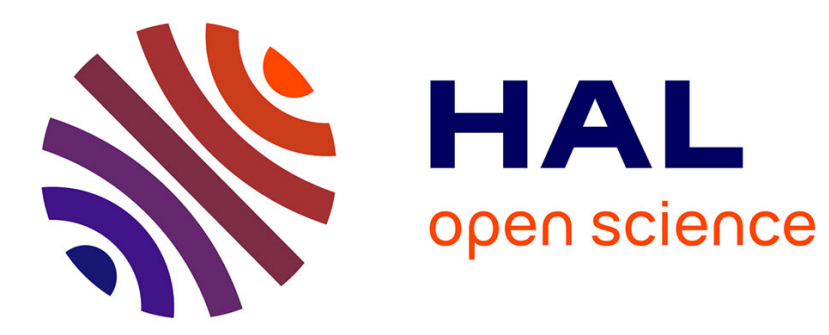

\title{
Feature Evaluation for Effective Bearing Prognostics.
}

Fatih Camci, Kamal Medjaher, Noureddine Zerhouni, Patrick Nectoux

\section{To cite this version:}

Fatih Camci, Kamal Medjaher, Noureddine Zerhouni, Patrick Nectoux. Feature Evaluation for Effective Bearing Prognostics.. Quality and Reliability Engineering International, 2012, pp.1-15. 10.1002/qre.1396 . hal-00798464

\section{HAL Id: hal-00798464 https://hal.science/hal-00798464}

Submitted on 8 Mar 2013

HAL is a multi-disciplinary open access archive for the deposit and dissemination of scientific research documents, whether they are published or not. The documents may come from teaching and research institutions in France or abroad, or from public or private research centers.
L'archive ouverte pluridisciplinaire HAL, est destinée au dépôt et à la diffusion de documents scientifiques de niveau recherche, publiés ou non, émanant des établissements d'enseignement et de recherche français ou étrangers, des laboratoires publics ou privés. 


\title{
Feature Evaluation for Effective Bearing Prognostics
}

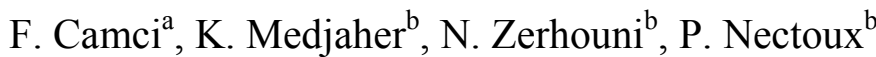 \\ ${ }^{\text {a }}$ IVHM Centre School of Applied Sciences Cranfield University, UK \\ ${ }^{\mathrm{b}}$ FEMTO-ST Institute, UMR CNRS 6174-UFC/ENSMM/UTBM, France
}

\begin{abstract}
Rolling element bearing failure is one of the foremost causes of breakdown in rotating machinery. It is not uncommon to replace a defected/used bearing with a new one that has shorter remaining useful life than the defected one. Thus, prognostics of bearing plays critical role for increased availability and reduced cost. Effective prognostics highly depend on the quality of the extracted features. Diagnostics is basically a classification problem, whereas the prognostics is the process of forecasting the future health states. The quality of the features for classification has been studied thoroughly. However, evaluation of the quality of features for prognostics is a relatively new problem. This paper presents an evaluation method for the goodness of the features for prognostics and presents results on bearings run until failure in a lab environment.
\end{abstract}

\section{Introduction}

Rolling element bearing failure is one of the foremost causes of breakdown in rotating machinery [1]. Bearing faults account for the $40 \%$ of motor faults according to the research conducted by Electric Power Research Institute (EPRI) [2]. Turbine engine bearing failures are the leading cause of class-A mechanical failures (loss of aircraft) [3]. Even one aircraft saved with prognostics would pay its development cost [4].

Bearing faults can be categorized as follows: 1) outer bearing race defects 2) inner bearing race defect 3) ball defects 4) cage (train) defect. There are also faults such as imbalance, misalignment, looseness, and debris contamination, which include high randomness related to environment and human error. Bearings are typically designed to have a life greater than the subsystem they are in. Failure initiation in bearings includes high randomness especially for failures related to debris contamination or mishandling.

Currently, defects of size much smaller than $6.25 \mathrm{~mm}^{2}$, which is commonly considered as a fatal failure size by industry standard [5], can be detected using diagnostics methods. However, failure detection forces machinery to shut down that causes tremendous time, productivity and capital loss. In addition, it is not uncommon to replace a defected/used bearing with a new one that has shorter remaining useful life than the defected one. For example, remaining useful life of a bearing with a newly detected defect may be substantially more than its $\mathrm{L}_{10}$ life, which is the life of $90 \%$ bearing population survival [1], [5]. Thus, identification of the most convenient time of maintenance after failure detection without reducing the safety requirements is crucial, which is possible with prognostics capability. Thus, bearing prognostics is very critical for effective operation \& management.

Each failure type causes a distinct signature in the vibration frequency [2] and vibration analysis is considered as the most reliable method in bearing failure detection [6]-[9]. However, it is often difficult to extract the failure signature due to the noise in the data 
especially in early stages of the failure [10]-[12]. Thus, several other sensor types such as current [13] and angular speed [14] have been used for bearing fault detection.

Prognostics is far more difficult task than diagnostics. In prognostics, failure progression should be modelled and forecasted in addition to the diagnostics. The prognostics methods can be broadly grouped into two categories: physics-based and empirical-based. Physics based methods analyze the physical nature of the system and failure as shown in Fig. 1 and have potential to lead to precise estimations if it can be modelled properly. However, reaching to perfect physical modelling is very difficult, if not impossible, especially for complex systems. Even though physics based prognostics models have been attempted for a variety of mechanical components with some success and might give better results than empirical-based models, they are much more expensive to implement. In addition, the replication of a physics-based method to slightly different equipment is prohibitive and intractable. Any small modification in the material or sub-component of the system will lead to need of remodelling. Physicsbased methods also have scalability problems.

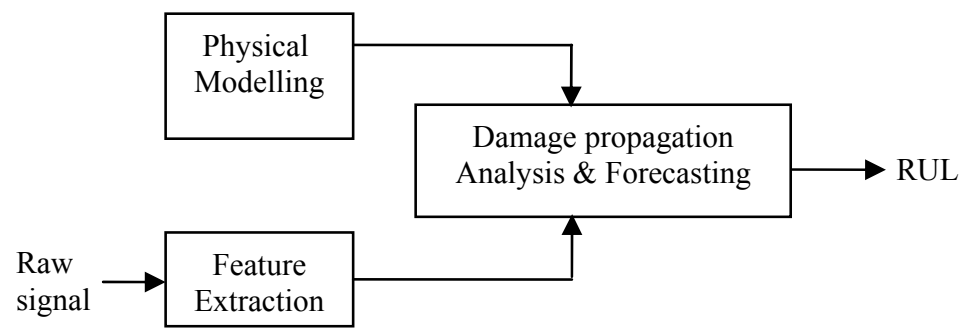

Fig 1: Physics based prognostics

In physics based prognostics, a deterministic fatigue crack propagation model based on Paris's formula, which relates the stress intensity to crack growth under fatigue stress, was presented for bearing prognostics in late 90s [1]. The model is enhanced by modification with a log-normal random variable [5]. Bearings are unique in failure progression since spall formation occurs as a result of thousands of small cracks rather than the propagation of a single dominant crack. Thus, traditional fatigue modelling cannot represent the failure progression perfectly [4].

Empirical prognostic methods can be grouped into three categories: First approach, evolutionary prognostic, involves trending of features combined with simplistic thresholds set from past experience and analysis of change rate from current condition to the known failure in feature space as shown in Fig 2. FFT, Wigner-Ville distribution, wavelet, Hilbert-Huang transform, blind source separation, statistical signal analysis are examples of the first group [15].

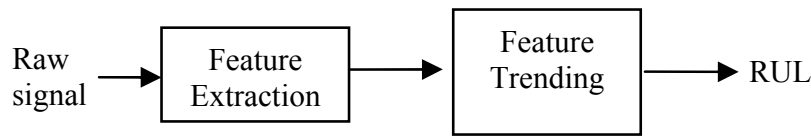

Fig 2: Evolutionary Based Empirical Prognostics

The complexity of the systems often require more processing for effective prognostics. Second approach in empirical prognostic methods is to utilize statistical regression models and/or computational intelligence methods such as Artificial Neural Network (ANN)-based [16], Genetic Algorithm (GA)-based [17], Fuzzy Logic (FL) based 
methods [18] to model known failure degradation paths in feature space. Thus, some valuable properties (features) of signals are extracted and used in intelligent systems for further processing as shown in Fig 3. Third approach, future state estimation, estimates a state vector that represents the equipment health condition from brand new to failure by employing subspace and non-linear dynamic methods as shown in Fig 4. These methods forecast the progression of health states of the machine from current state estimated by diagnostician to the failure state by employing transition probabilities between states and time spent in each state. Hidden Markov Model is the most commonly known state based prognostics method [19]-[23].

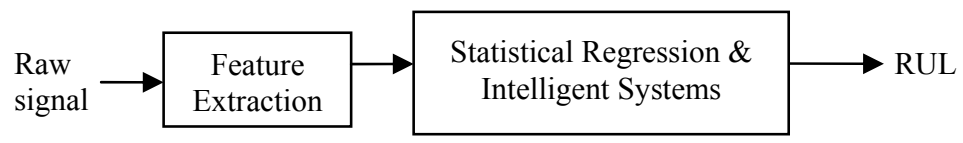

Fig 3: Regression/Computational Intelligence Based Empirical Prognostics

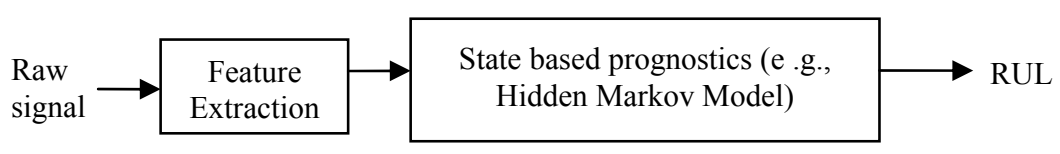

Fig 4: State Based Empirical Prognostics

As can be seen from the figures above, feature extraction is the common step in all types of prognostic approaches and one of the most critical steps in diagnostics and prognostics. Diagnostic and prognostic methods use the extracted features as input and their effectiveness rely on the features' representation capability to the failures and their progression. The goodness (quality) of the features affects the complexity of the diagnostic and prognostic methods. Features that represents healthy, close to failure machinery and their progression perfectly may lead to very simple diagnostic and prognostic methods. On the other hand, very complex diagnostic and prognostic methods using features that are ineffective in representation of failure and failure progression may lead to poor results. Thus, extraction of effective features is a prerequisite for effective diagnostics and prognostics.

Diagnostics is a classification problem, whereas the prognostics is the process of forecasting the future health states. The goodness of the features for diagnostics is basically a measure of separability between data from healthy and faulty equipment. Good separability indicates that samples from different classes (i.e., healthy and faulty) are far apart from each other and samples from the same class are close to each other. Within class separability (parameters $a$ and $b$ in Fig 5) and between class separability (parameter $\mathrm{c}$ in Fig 5) are used to quantify the separability. Many class separation metrics have been reported in the literature [24]-[25]. These metrics focus on static classes; do not consider progression from one class to another. One feature may be good at separation of the classes, but not at representation of progression from one class to another. For example, separability measure $\left(\mathrm{S}_{2}\right)$ of feature $2(\mathrm{~F} 2)$ is higher than in separability measure $\left(\mathrm{S}_{1}\right)$ of feature $1(\mathrm{~F} 1)$ in Fig 5 . However, this does not mean that $\mathrm{F} 2$ is better in representing the failure progression. As seen from the figure, failure progression in F2 involves higher variation. Thus, a new quality measure should be employed for prognostics, which is a relatively new problem. 

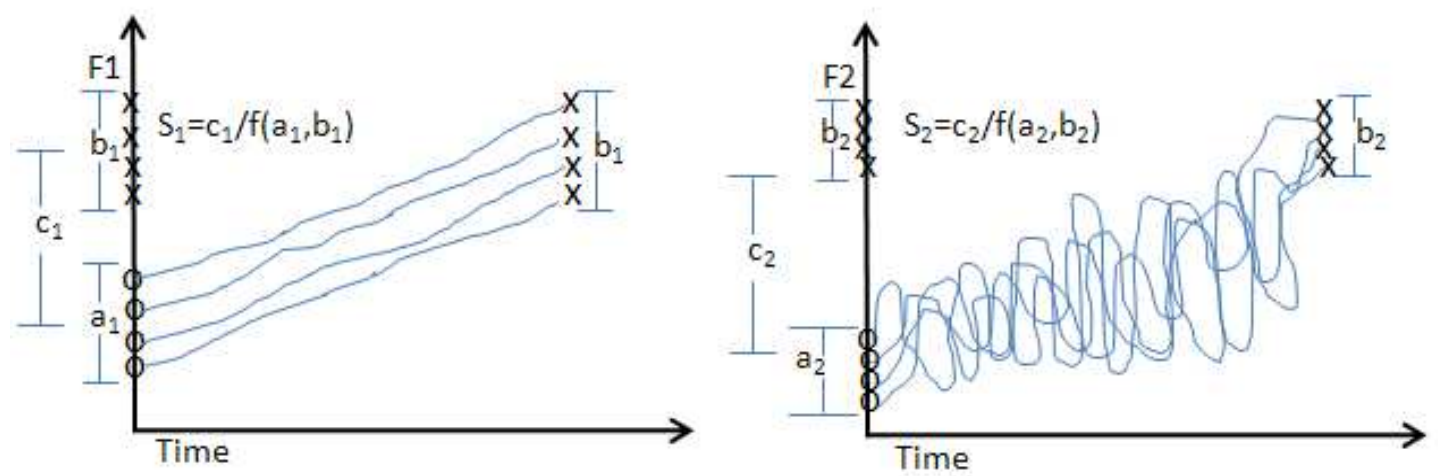

Fig 5: Feature quality for diagnostics and prognostics

This paper presents an evaluation method for the goodness of the features for prognostics. An effective feature evaluation method will achieve the selection of best features, which is critical for obtaining better prognostics results. Organizations may focus on the development of core prognostics methods after ensuring that the selected features are the best ones for prognostics. The feature evaluation method is applied to bearings that were run until failure in a lab environment. Section II presents the quantification metric for the quality evaluation of features for prognostics. Section III presents results and experiments. Section IV concludes the paper.

\section{Goodness of Failure Progression Representation Metric}

This section presents a metric that evaluates the goodness of features in failure progression representation. A good feature for prognostics should be monotonically non-decreasing or non-increasing for all samples. Formal definition of monotonically non-increasing and non-decreasing function is given below.

Monotonically non-increasing or non-decreasing: Mathematically, a function $f$ is called monotonically increasing (monotonically non-decreasing), if for all $x$ and $y$ such that $x \leq y$ one has $f(x) \leq f(y)(f(\mathrm{y}) \leq f(\mathrm{x}))$. Examples of monotonically non-increasing and non-decreasing functions are given in Fig 6.
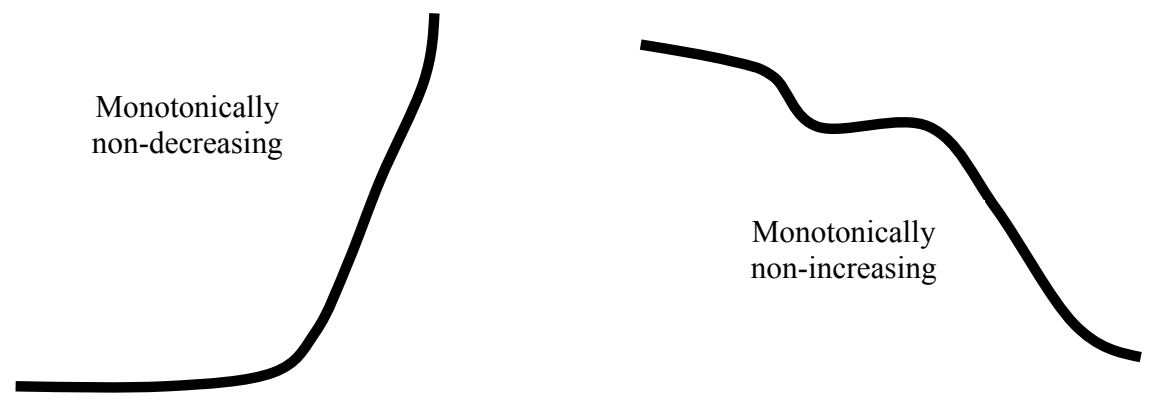

Fig. 6. Examples of monotonically non-decreasing and non-increasing functions

It may be trivial to check the monotonicallity for a single failure progression sample by analyzing the difference between consecutive points. When all the difference values are greater (less) than or equal to 0, then the function is defined as non-decreasing (nonincreasing). However, monotonicallity over all samples representing failure progression should be considered rather than individual analysis of samples. Example of several samples representing failure progression is displayed in Fig. 7. As seen from the figure, 
the time is segmented for effective analysis of the failure progression. The effectiveness of a feature to represent the failure progression is calculated as the average separability of segments as represented in (1). The higher the total separability value (S) is, the better representation of the failure progression. Thus, the goal is to find the feature that has the highest $\mathrm{S}$ value.

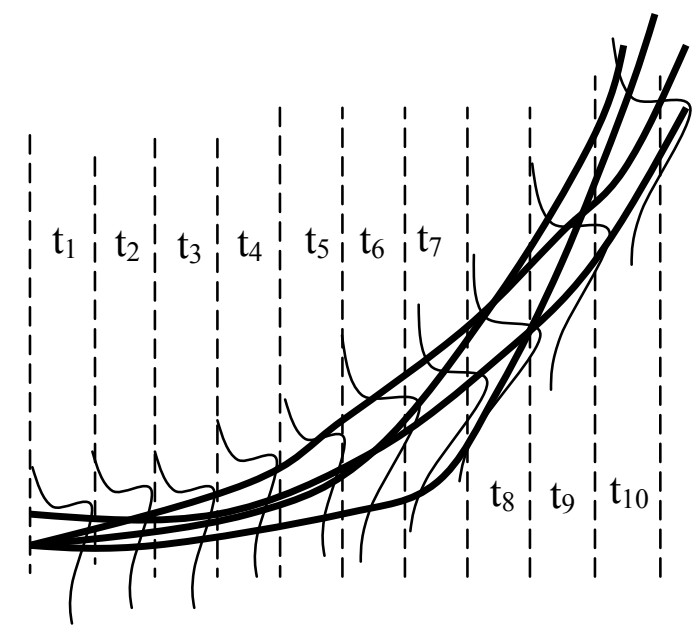

Fig. 7. Failure progression for multiple samples

$$
S=\frac{\sum_{t=1}^{T} s_{t}}{T}
$$

S: Average separability value

$\mathrm{s}_{\mathrm{t}}$ : Separability at time $\mathrm{t}$

$\mathrm{T}$ : Total number of time segments

The distribution of the data points from different samples in each time segment should be used to measure the separability at a given time segment. $L$ represents the distance between $25^{\text {th }}$ and $75^{\text {th }}$ percentiles. The ratio of the length of the non-overlapped portion (called a) to $L$ is a measure of the separability $(\mathrm{a} / \mathrm{L})$. When the separation is low as in Fig 8.A, a/L ratio will be close to 0 . When the separation is high as in Fig $8 . \mathrm{B}, \mathrm{a} / \mathrm{L}$ becomes closer to 1 . When there is no overlap between 25-75 percentiles of the distributions $(\mathrm{a} / \mathrm{L}=1)$, there exist two different possibilities. In the first one, there is some overlap within data greater than $75^{\text {th }}$ percentile or less than $25^{\text {th }}$ percentile as shown in Fig.8.C. The second one represents complete separation as shown in Fig. 8.D. When $a / L$ becomes 1 , then the ratio of number of data points causing overlap to the total number of data points in the distribution is subtracted in separability calculation. The separability calculation is formulated in (2).

$$
\begin{aligned}
& s_{t}=\frac{a}{L}-\frac{\chi}{N_{t}} \\
& \chi= \begin{cases}0 & \text { if } \frac{\mathrm{a}}{\mathrm{L}} \neq 1 \\
\alpha & \text { if } \frac{\mathrm{a}}{\mathrm{L}}=1\end{cases}
\end{aligned}
$$


$\alpha$ : Number of samples overlapping with the distribution in consecutive time frame $N_{t}$ : Number of samples in time segment $t$

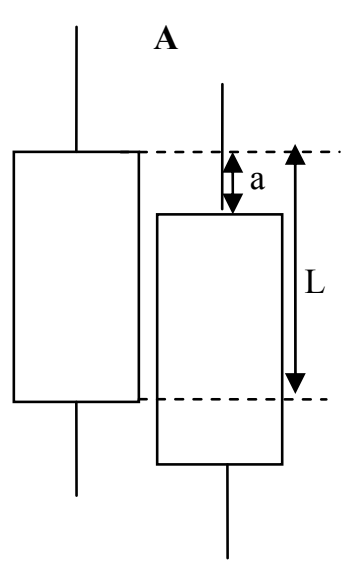

$\mathrm{s}_{1}=\mathrm{a} / \mathrm{L}$

Low

separation, low s

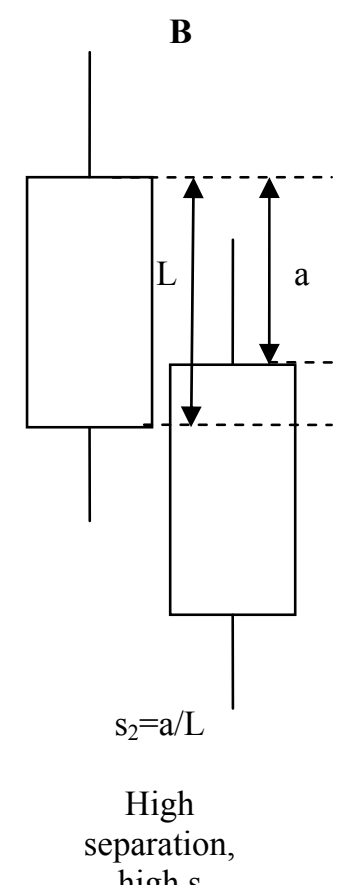

high s

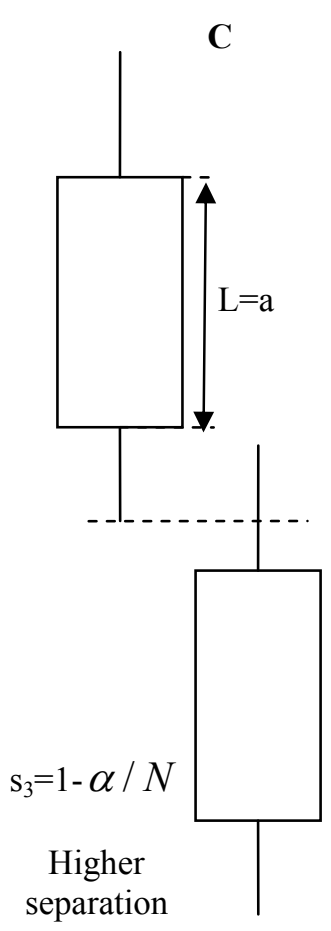

Fig 8. Illustration of increase and decrease

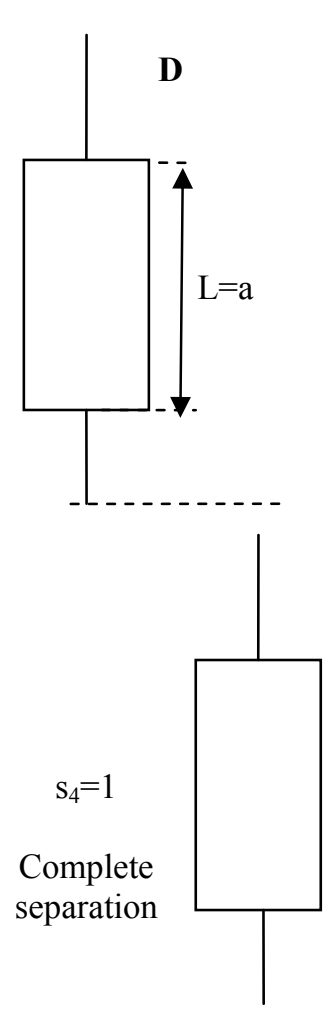

\section{Experiments \& Results}

The presented feature evaluation metric will be applied to real data obtained from a bearing experiment set. Two applications are discussed below.

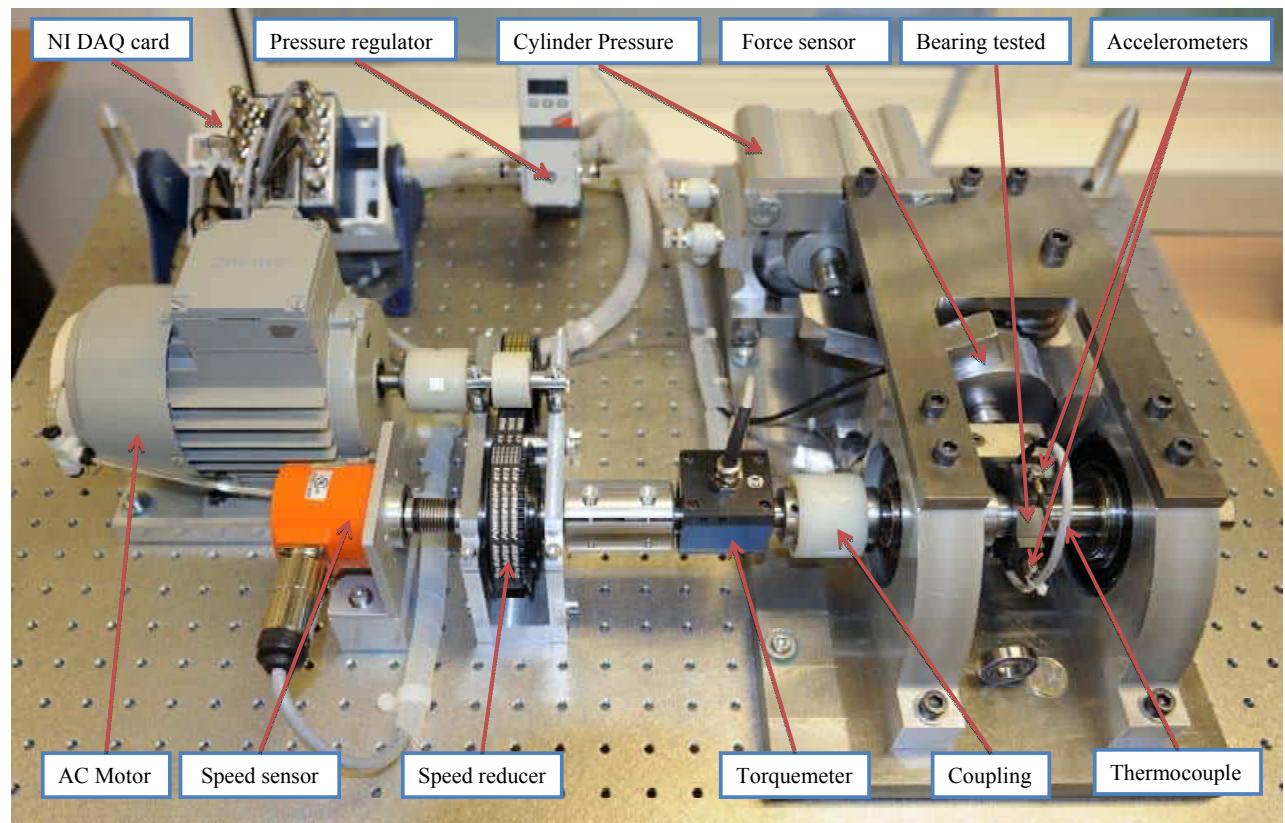

Fig 9. PRONOSTIA experimental Platform 
1. Experimental Setup: The accelerated bearing life test bed is called PRONOSTIA (displayed in Fig. 9), which it is an experimentation platform dedicated to test and to validate bearing health assessment, diagnostic and prognostic. In the present experimental setup a natural degradation process of bearings is performed. During the experiments any failure types (inner race, outer race, ball, or cage) or their combinations could occur. This is allowed in the system to better represent a real industrial situation.

The experimental platform PRONOSTIA is composed of two main parts: a first part related to the speed variation and a second part dedicated to load profiles generation. The speed variation part is composed of a synchronous motor, a shaft, a set of bearings and a speed controller. The synchronous motor develops a power equal to $1.2 \mathrm{~kW}$ and its operational speed varies between 0 and $6000 \mathrm{rpm}$. The second part is composed of a hydraulic jack connected to a lever arm allowing to create different loads on the bearing mounted on the platform for degradation.

A pair of ball bearings is mounted on one end of the shaft to serve as the guide bearings and a NSK6307DU roller ball bearing is mounted on the other end to serve as the test bearings. The transmission of the movement between the motor and the shaft drive is coupled by a rub belt.

Two high frequency accelerometers (DYTRAN 3035B) are mounted horizontally and vertically on the housing of the test roller bearing to pick up the horizontal and the vertical accelerations. In addition, the monitoring system includes one temperature probe (of type PT100) to record the temperature of the tested bearing. A speed sensor and a torque sensor are also available on the PRONOSTIA platform. The set of sensors installed on the platform allow the user to know at each time the current operating conditions of the experiment. A data acquisition card (NI DAQCard-9174) is also used, which allows the integration of three modules: the first includes the two acceleration sensors, the second is for the temperature probe and the third for the torque transducer. The monitoring data are transmitted via an USB 2.0 link to a dedicated computer where they are stored. The data acquisition software is programmed by using a LabView interface. The readings can be directly taken from the digital readout on the analyzer, graphical representation of the data can be displayed on the screen and finally, the data can be analyzed online or offline during later processing. The sampling frequency of the NI DAQCard-9174 data acquisition card is set to $25600 \mathrm{~Hz}$ and the vibration data provided by the two accelerometers are collected every 1 second. Each record is stored in a matrix format where the following parameters are defined: the time, the horizontal acceleration, the vertical acceleration, the temperature, the speed and the torque.

The bearing operating conditions are determined by instantaneous measures of the radial force applied on the bearing, the rotation speed of the shaft handling the bearing and of the torque inflicted to the bearing. Thus, three sensors are used: a load cell and its transducer amplifier, an incremental encoder and its analogue signal converter, and a torque transducer with its converter. With this experimental platform, several types of profile can be created by varying the load and the speed. This is very important as it allows simulating constant as well as variable operating conditions for bearing's degradation. For the measurement of the degradation's characteristics during the test, the bearing starts from its nominal operating mode until its faulty operating mode or state. The bearing's behaviour is captured during its whole degradation process by using the different sensors. 
2 Extracted features: 12 different features are extracted to be used for failure progression (maximum, mean, standard deviation, skewness, kurtosis, root mean square error (RMS), crest factor and highest frequency). RMS and crest factor are applied in three different ways. The first one involves raw data. The other two pre-process the raw data by applying outlier detection with different levels using low pass and high pass filters. Thus, total of 36 features are analyzed for bearing prognostics in this paper.

After analyzing the data, two different types of degradation are detected. The first type occurs from the initial use of the bearing until the failure reaches a severity level. The second type of degradation includes the data working in a failure state. The first type is relatively smooth compared to the second type. The second type of degradation includes more variability and is more unpredictable. Both degradation types are analyzed in this paper and displayed in Fig. 10.

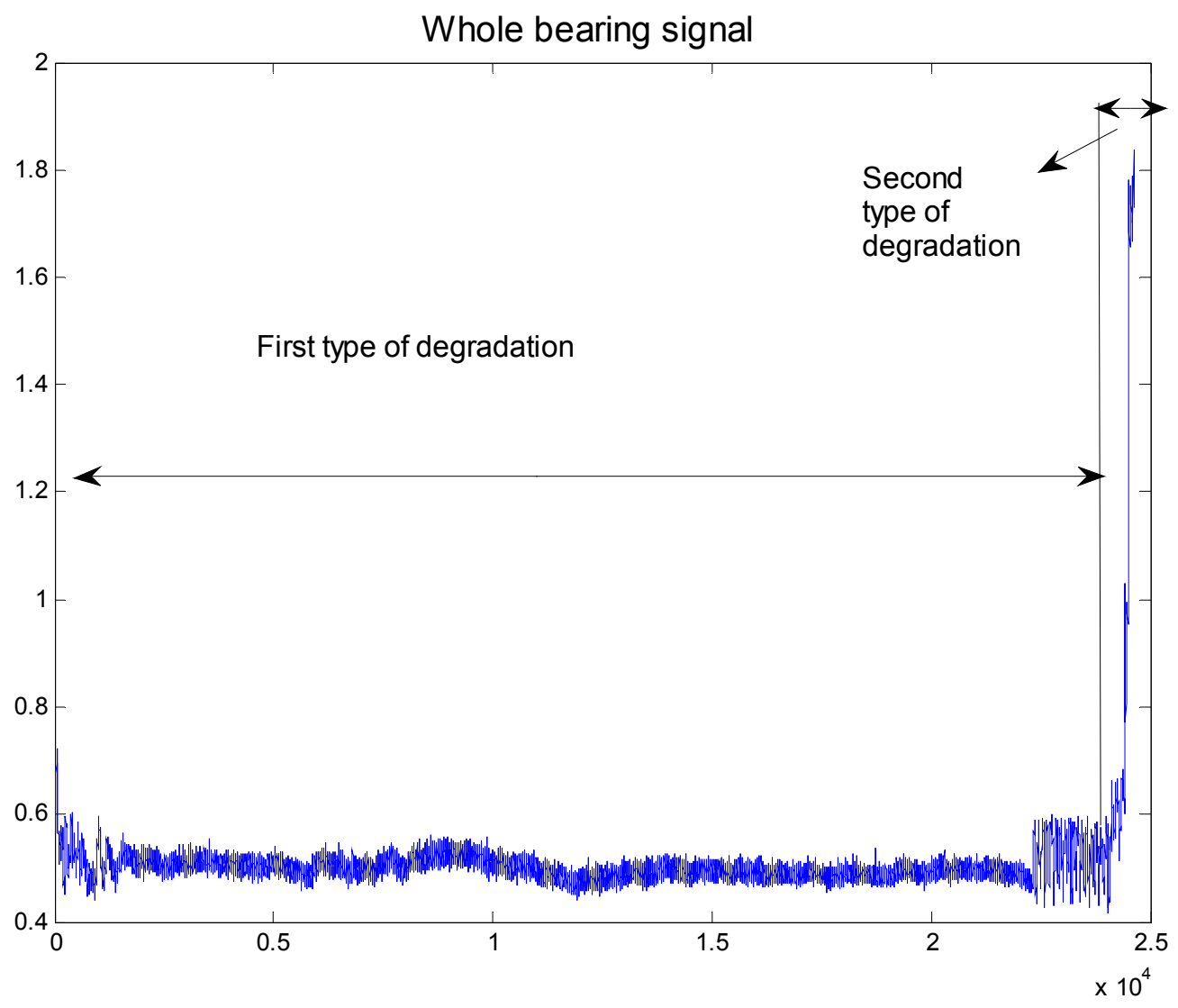

Fig. 10: Vibration signal obtained for whole life of a bearing 

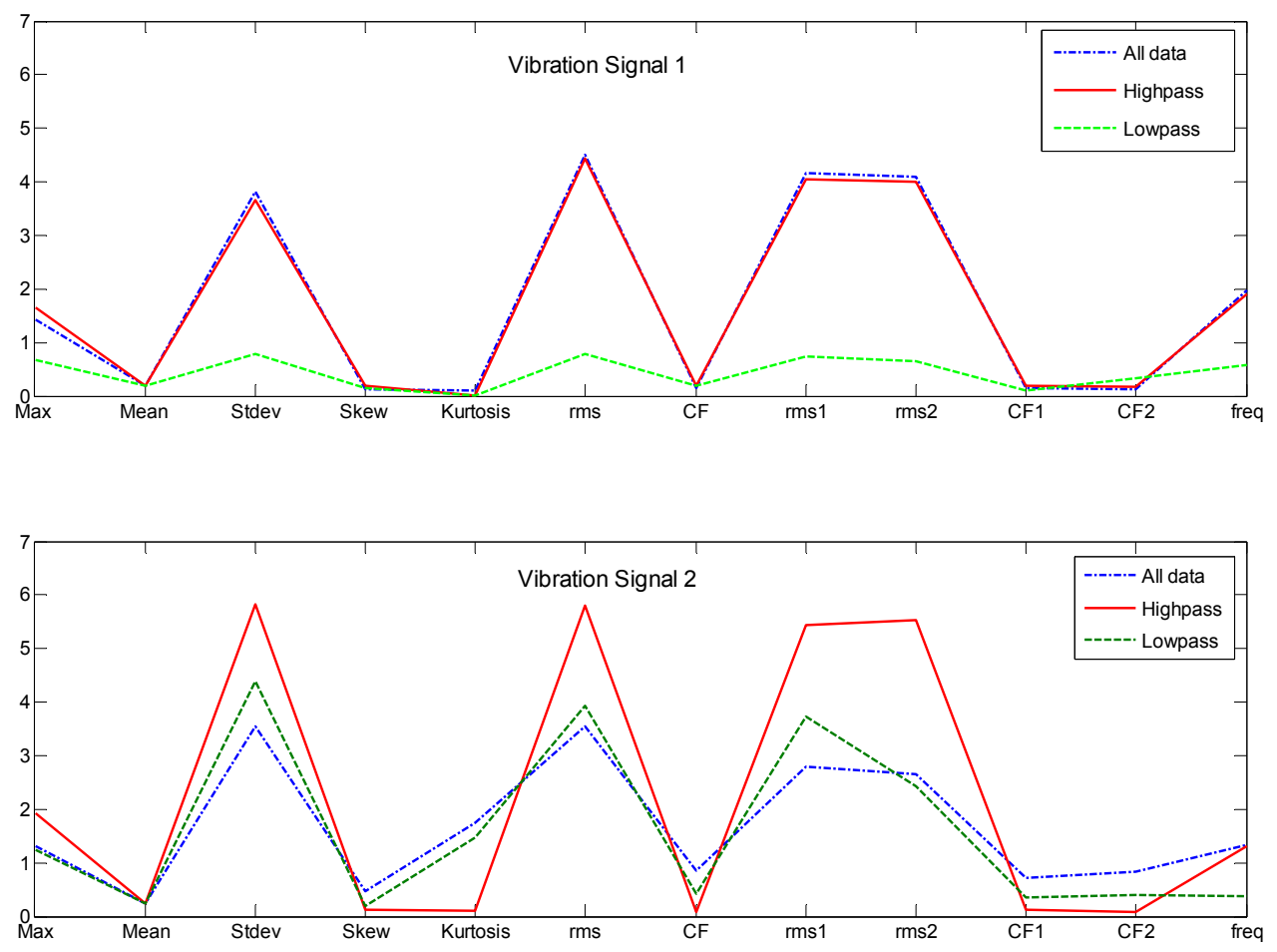

Fig. 11. Separability measure for selected features

Fig. 11 displays the separability values obtained for all features for two vibration signals from the first degradation type. The following analysis can be obtained from the figure.

1. Standard deviation and RMS (RMS, RMS1, and RMS2) are the best features with highest separability values for both vibration signals.

2. The failure signature is hidden within the high frequency data because when low pass filter is applied the separability values decrease even for the best features. Fig. 12 that displays the frequency time amplitude diagram for bearing fault justifies the statement above. The effects of the failure are seen in high frequencies.

3. The separability measure is increased by applying high pass filter in vibration signal 2 . The effects of failure occur in high frequencies in vibration signal 2 and high pass filter is able to filter the unnecessary data successfully. However, no significant difference can be observed in vibration signal 1 when high pass filter is applied. The effect of bearing failure on vibration signal 1 is either spread over all frequencies or the high pass filter needs to be optimized further for more effective results. Since the main focus of this paper is not to extract the best features, but to create a methodology for feature evaluation in prognostics; the filtering methods are not thoroughly optimized.

4. Vibration signal 2 (horizontal vibration) is more sensitive to the bearing failure compared to vibration signal 1 (vertical). This result 
is logical due to the experimental setup. It is acknowledged by the experts that the horizontal vibration is more sensitive to the failure. Maximum separability value of vibration signal 2 is around 6 whereas it is around 4 for vibration signal 1 . If one needs to select only one vibration sensor; it is recommended to have the second vibration sensor based on these results.

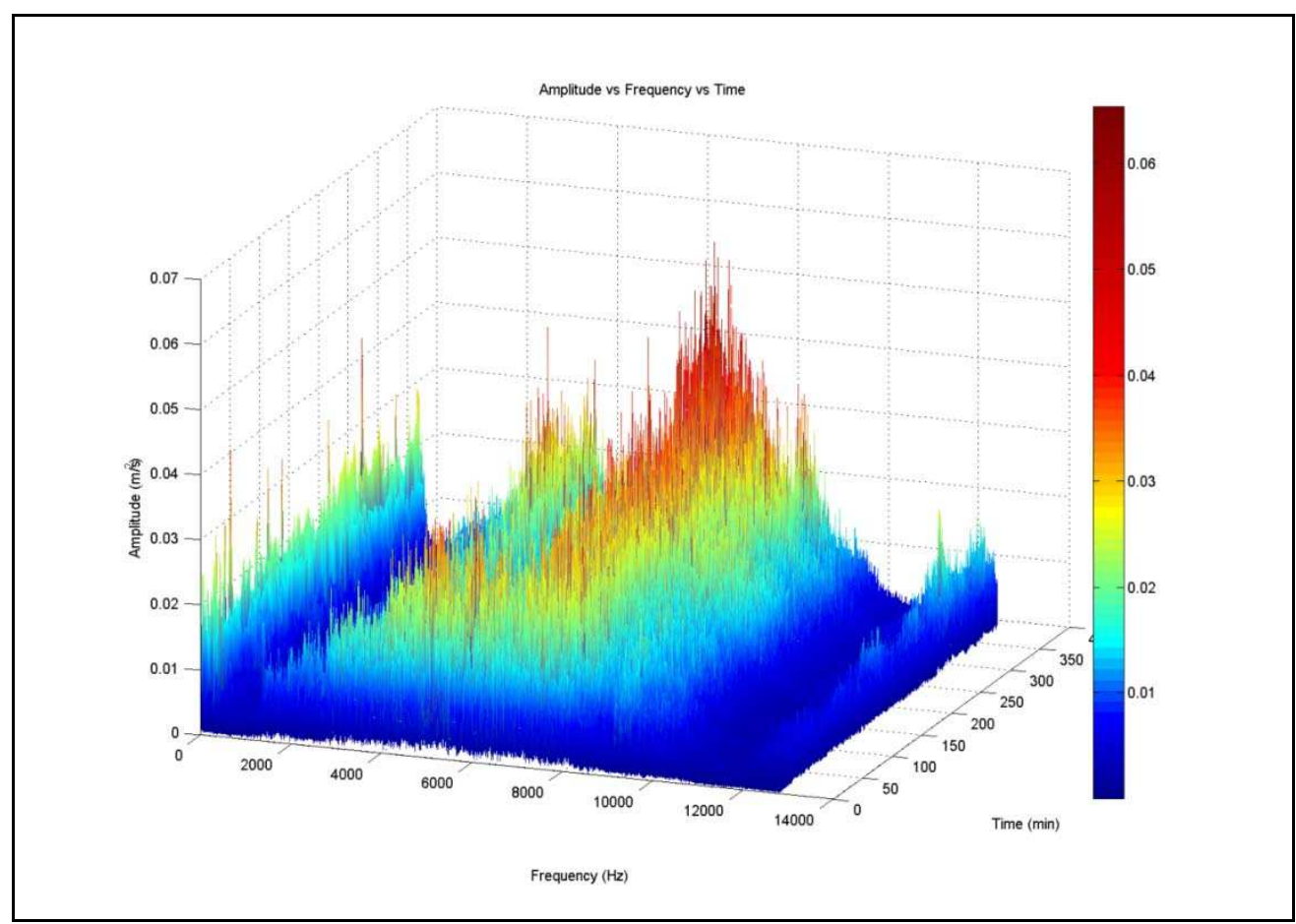

Fig 12: Frequency time amplitude for bearing fault analysis

Fig 13 displays two good (RMS and standard deviation); two bad features (Skewness and crest factor) for prognostics. As you can see from the figures, failure progression can be seen in the features with high separability measure. 

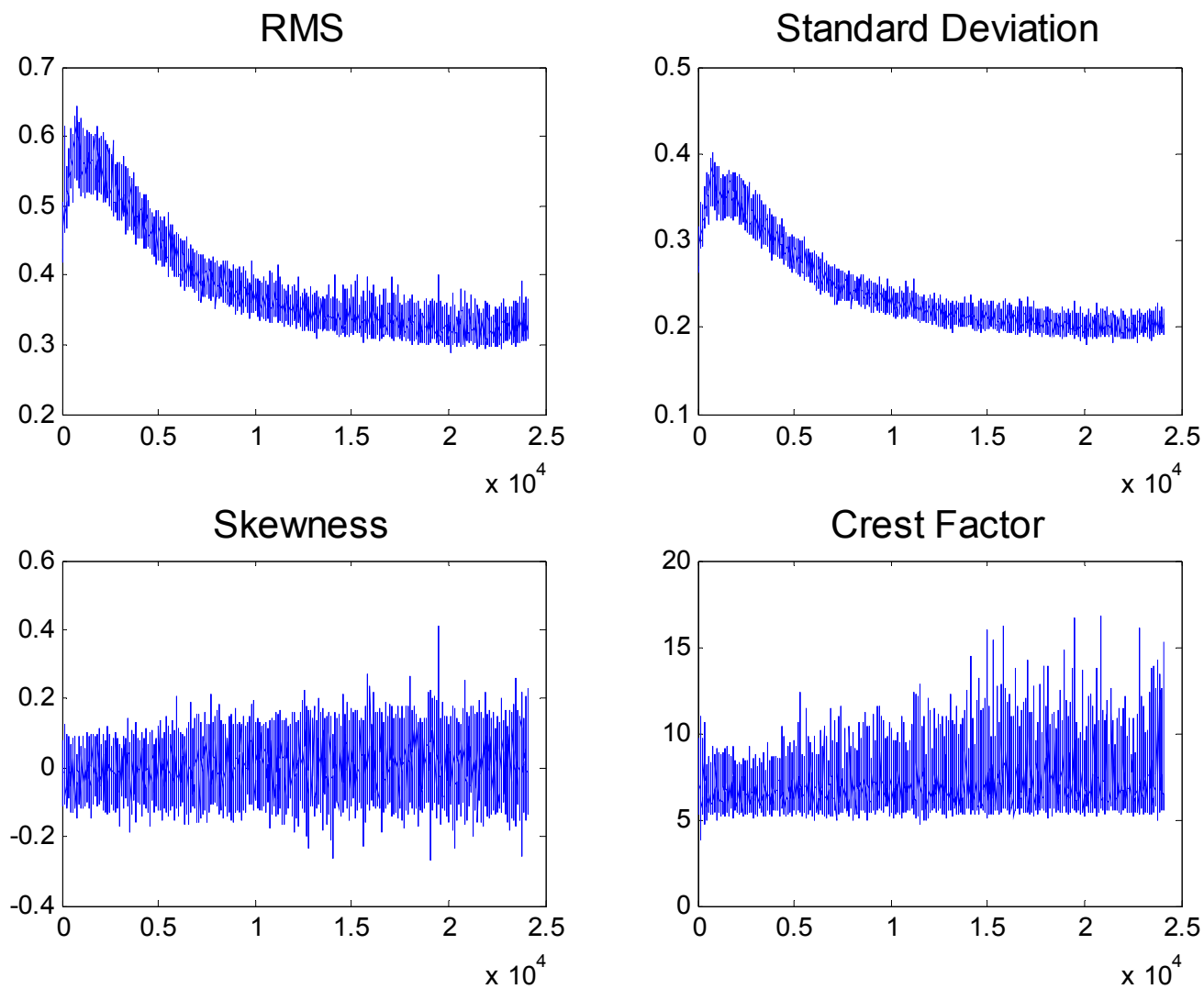

Fig 13: Illustration of good and bad features

Fig 14 displays the standard deviation of both vibration signals. As seen from the figure the failure progression in vibration signal 2 is clearer. However, it is not always easy and possible to visually observe the failure progression. Thus, quantification metric for representation ability of failure progression is important. 

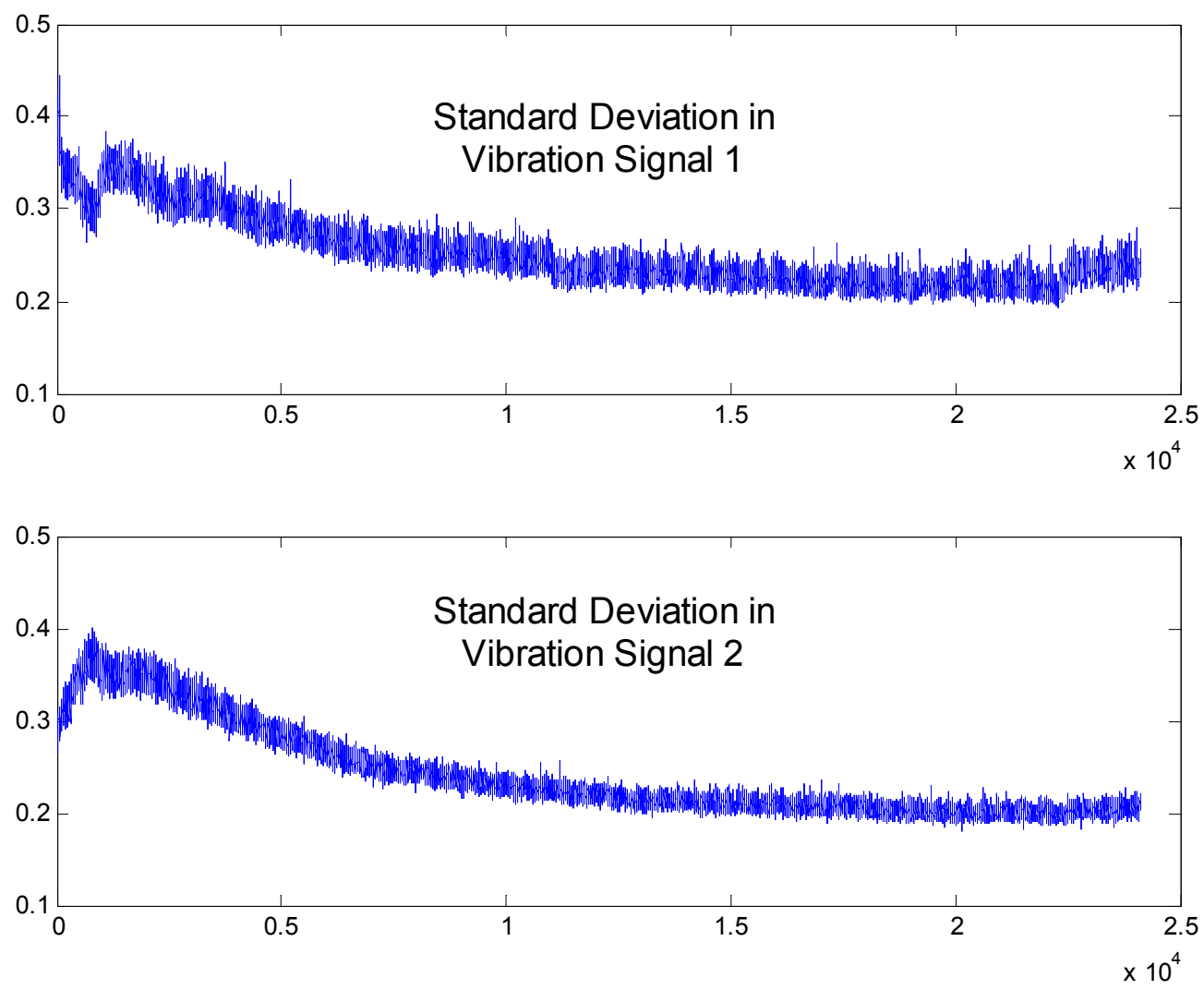

Fig 14: Illustration of good and bad features

Fig 15 displays the separability values of second type of degradation. Similar results are obtained with the first type of degradation. Due to high variability, the effects of failure are seen in high range of frequencies. Thus, analysis of raw data without filtering gives better results. In this type of degradation, the effects of the failure are very clear and can be seen in both vibration sensors in similar degrees. Fig 16 displays the examples of good and bad features for failure degradation. Standard deviation and RMS are the good examples, whereas skewness and crest factor are the bad examples.

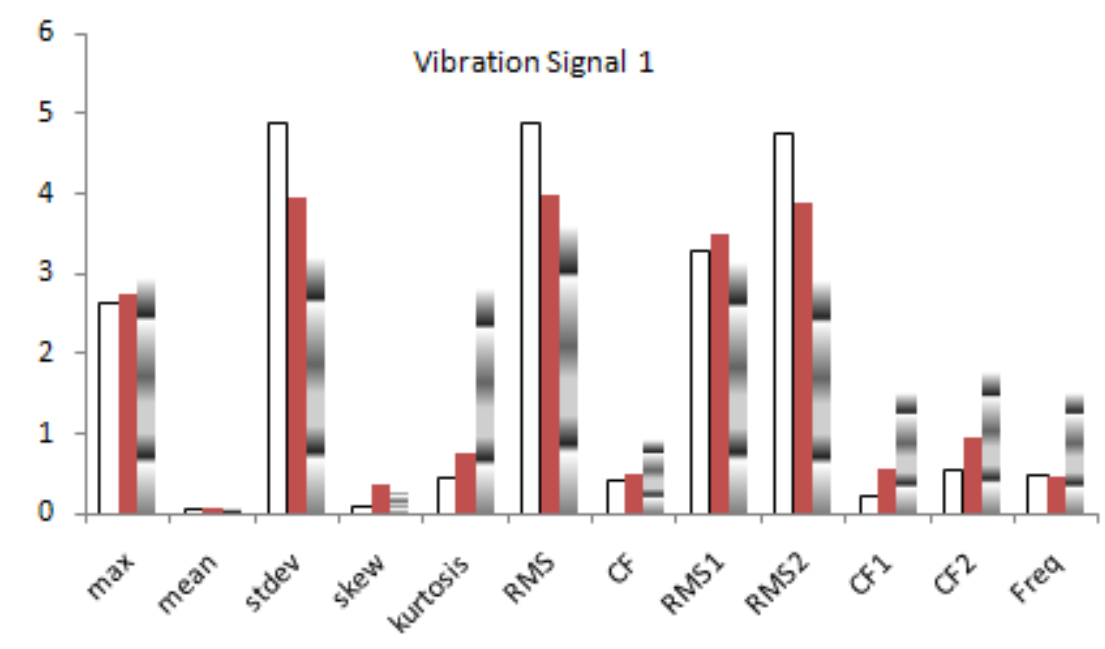




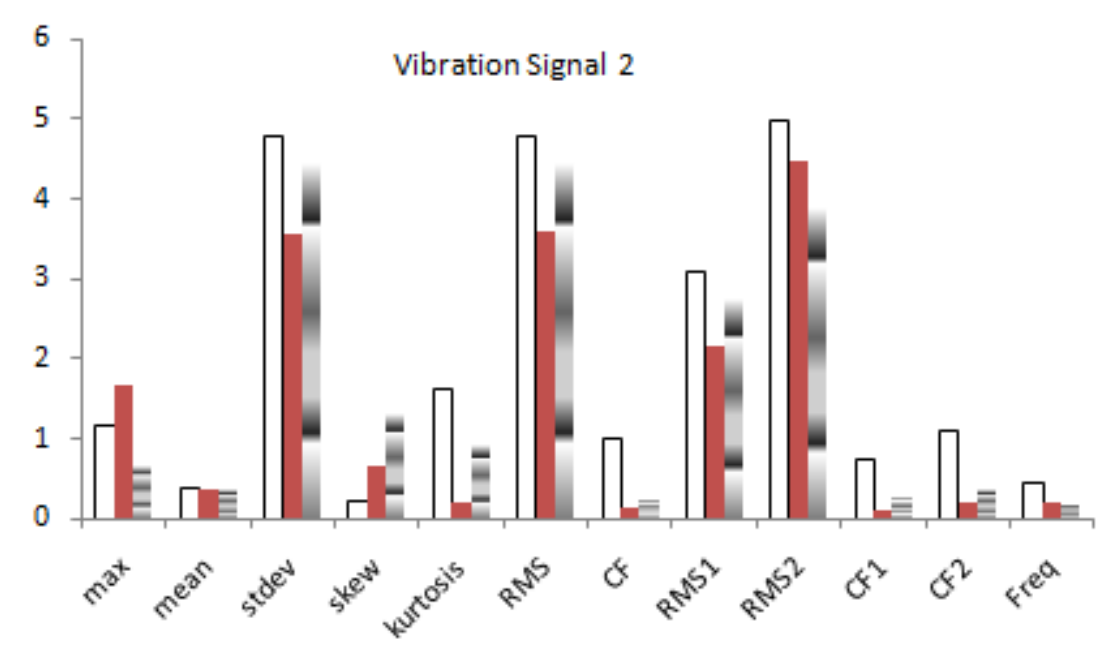

Fig 15: Separability values for the second type of degradation
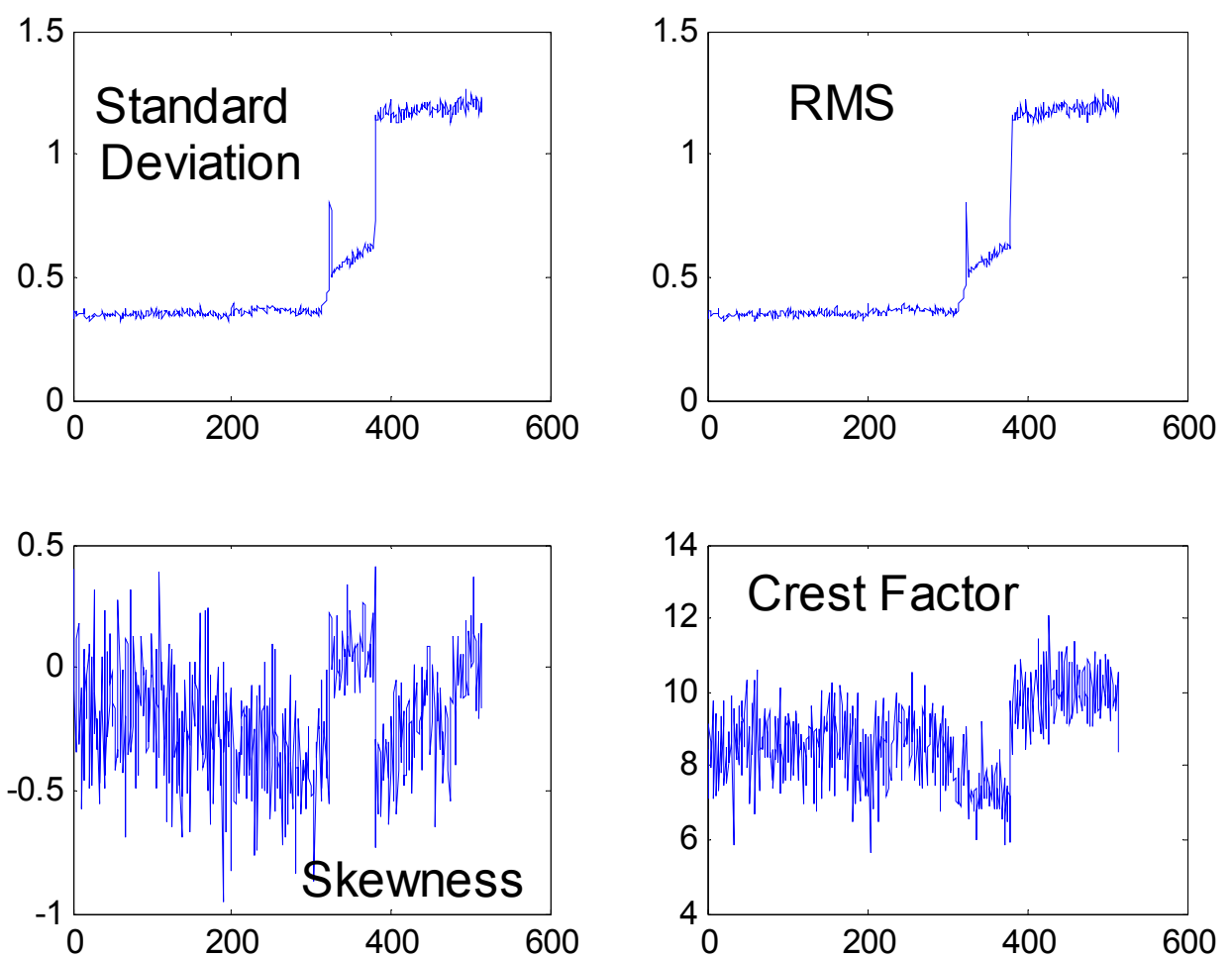

Fig 16: Examples of good and bad features for failure degradation

\section{Conclusion}

The quality of the features is critical for effective diagnostics and prognostics. Diagnostics is the identification of an existing failure, whereas prognostics is the forecasting the time of failure before it occurs. Evaluation of the quality of features in diagnostics has been studied extensively since diagnostics is essentially a classification problem. On the other hand, evaluation of the features for prognostics is a new problem. This paper presents quantification metric for evaluation of the quality 
of features. The presented metric is applied to features extracted from bearing vibration data collected. Bearings were run until failure in a lab environment. 12 features are extracted for raw, high pass filtered, and low pass filtered data. The results obtained from the presented metric are very promising and justified with analysis of the bearing failure analysis.

\section{References}

[1] Li Y., Billington S., Zhang C., Kurfess T., Danyluk S., Liang S., "Adaptive Prognostics For Rolling Element Bearing Condition”, Mechanical Systems and Signal Processing 13(1), 103-113, 1999

[2] Enzo C.C. L., Ngan H. W., "Detection of Motor Bearing Outer Raceway Defect by Wavelet Packet Transformed Motor Current Signature Analysis", IEEE Transactions on Instruments and Measurement, 59(10), 2683-2690, 2010

[3] Wade Richard A. "A Need-focused Approach to Air Force Engine Health Management Research" Health Management Research IEEE Aerospace Conference Big Sky, Montana, 2005

[4] Marble S., Morton B.P., "Predicting the Remaining Useful Life of Propulsion System Bearings", Proceedings of the 2006 IEEE Aerospace Conference, Big Sky, MT, USA, 2006

[5] Li Y., Kurfess T. R., Liang S. Y., "Stochastic Prognostics For Rolling Element Bearing”, Mechanical Systems and Signal Processing, 14(5), 747-762, 2000

[6] Zhang B., Sconyers C., Orchard M., Patrick R., Vachtsevanos G., "Fault Progression Modeling: An Application to Bearing Diagnosis and Prognosis", Proceedings of American Control Conference, MD USA, 2010

[7] Davaney M., Eren L., "Detecting Motor Bearing Faults", IEEE Instrumentation \& Measurement Magazine, 30-50, 2004

[8] McFadden P.D., Smith J. D., "Vibration monitoring of rolling element bearings by the high frequency resonance technique - a review", Tribology International, 17, 3-10, 1984

[9] Tandon N., Choudhury A., "A review of vibration and acoustic measurement methods for the detection of defects in rolling element bearings", Tribology International, 32, 469-480, 1999

[10] Su W., Wang F., Zhu H., Zhang Z., Guo Z., "Rolling element bearing faults diagnosis based on optimal Morlet Wavelet filter and autocorrelation enhancement", Mechanical Systems and Signal Processing, 24, 1458-1472, 2010

[11] Bozchalooi I. S., Liang M., "A joint resonance frequency estimation and in-band noise reduction method for enhancing the detectability of bearing fault signals", Mechanical Systems and Signal Processing 22, 915-933, 2008

[12] He W., Jiang Z. N., Feng K., "Bearing fault detection based on optimal wavelet filter and sparse code shrinkage", Measurement, 42, 1092-1102, 2009

[13] Immovilli F., Bellini A., Rubini R., Tassoni C., "Diagnosis of Bearing Faults in Induction Machines by Vibration or Current Signals: A Critical Comparison", IEEE Transactions on Industry Applications, 46(4), 1350-1359, 2010

[14] Renaudin L., Bonnardot F., Musy O., Doray J. B., Remond D., "Natural roller bearing fault detection by angular measurement of true instantaneous angular speed", Mechanical Systems and Signal Processing 24, 1998-2011, 2010

[15] Rafiee J., Rafiee M. A., Tse P. W., "Application of mother wavelet functions for automatic gear and bearing fault diagnosis", Expert Systems with Applications, $37,4568-4579,2010$ 
[16] Paya B.A., Esat I. I., Badi M. N. M., "Artificial neural network based fault diagnosis for rotating machinery using wavelet transforms as a pre-processor", Mechanical Systems and Signal Processing, 11(5), 751-765

[17] Samanta B., Gear fault detection using artificial neural network \& support vector machine with genetic algorithms, Mechanical Systems and Signal Processing, 18(3), 625-644, 2004

[18] Saravanan N., Cholairajan S., Ramachandran K. I., "Vibration-based fault diagnosis of spur bevel gear box using fuzzy technique", Expert systems with applications, 35(3), 1351-1366

[19] Ocak H., Loparo K. A., Discenzo F. M., "Online Tracking of bearing wear using wavelet packet decomposition and probabilistic modelling: A method for bearing prognostics", Journal of Sound and Vibration, 302, 951-961, 2007

[20] R. B. Chinnam, P. Baruah, Autonomous diagnostics and prognostics in machining processes through competitive learning-driven HMM-based clustering, International Journal of Production Research, 47 (23), 2009, 6739 - 6758.

[21] M. Dong, D. He, A segmental hidden semi-Markov model (HSMM) -based diagnostics and prognostics framework and methodology, Mechanical Systems and Signal Processing, 21, 2007, 2248-2266

[22] Camci, R. B. Chinnam., "Health-State Estimation and Prognostics in Machining Processes", IEEE Transactions on Automation Science and Engineering, 7(3), 2010, 581-597

[23] P. Baruah and R. B. Chinnam, HMMs for diagnostics and prognostics in machining processes, "International Journal of Production Research", 43(6), 2005, 1275-1293

[24] Calinski R. B. and Harabasz J., A Dendrite Method for Cluster Analysis, Comm. in Statistics, 3, 1974, 1-27

[25] Eker O. F., Camci F, Guclu A., Yilboga H., Sevkli M., Baskan S.,"A Simple State- based Prognostic Model for Railway Turnout Systems", IEEE Transactions on Industrial Electronics, Vol. 58, No. 5, May 2011, 1718-1726 